\title{
SAXS studies of X-ray induced disulfide bond damage: Engineering high- resolution insight into a low resolution technique
}

\author{
T Stachowski ${ }^{1}$, E Snell ${ }^{1}$, E Snell ${ }^{2}$ \\ ${ }^{1}$ Hauptman Woodward Institute, Buffalo, NY, ${ }^{2}$ Hauptman-Woodward Medical Research \\ Institute/BioXFEL \\ tstachowski@hwi.buffalo.edu
}

\begin{abstract}
A significant problem in biological X-ray crystallography is the radiation chemistry caused by the incident X-ray beam. This produces both global and site-specific damage. Global damage manifests itself in the decay of the diffraction pattern and data processing parameters. Site specific damage targets glutamates, aspartates, methionines, and disulfide bonds. This damage can misdirect the biological interpretation of the structural models produced. Cryo-cooling crystals has been successful in mitigating damage but not eliminating it altogether; however, cryocooling can be difficult in some cases and has also been shown to limit functionally relevant protein conformations. Due to this, there has been an interest in the return to near-physiological temperature studies. The doses used for Xray crystallography are typically in the kGy to MGy range. X-rays are used therapeutically at significantly lower doses. While, disulfide bonds are among the most significantly affected species in proteins in the crystalline state at both cryogenic and higher temperatures, there is limited information on their response to low X-ray doses. In this work we engineered a protein that dimerizes through a susceptible disulfide bond to relate the radiation damage processes seen in crystals to those closer to physiologic conditions. This approach enables a low-resolution technique, small angle X-ray scattering (SAXS), to detect and monitor a residue specific process. We monitored monomerization with SAXS detecting structural impact with doses a fraction of that required for crystallographic studies. A dose dependent fragmentation of the engineered protein was seen that can be explained by a dimer to monomer transition, from disulfide bond cleavage. This supports the crystallographically derived mechanism and demonstrates that results obtained crystallographically, can be usefully extrapolated to physiologic conditions. Fragmentation was $\mathrm{pH}$ dependent, providing information on mechanism and pointing to future routes for investigation and potential mitigation. The engineered protein approach generating a large-scale change through a site-specific interaction represents a promising tool for advancing radiation damage studies under solution conditions.
\end{abstract}

Acta Cryst. (2020). A76, a4 